\title{
Reparación endovascular de la aorta torácica, resultados a largo plazo
}

\author{
Luis C. Hernández M., ${ }^{1,2}$, Juan R. Bombin F. ${ }^{1,2}$, Alejandro J. Kotlik A. ${ }^{1,2}$, Angela G. Poblete S. ${ }^{1,2}$
}

\section{Endovascular repair of the thoracic aorta, long-term results}

Introduction: The pathological injuries of the descending thoracic aorta, represent an implicit threat to life, and are potentially treatable by endovascular repair. Aim: To evaluate the results of endovascular repair of the descending thoracic aorta (TEVAR) in the medium and long term. Material and Method: Observational, retrospective and descriptive study, where TEVAR is analyzed, over a period of 10 years (2009 to 2019), at the Dr. Eduardo Pereira Hospital in Valparaíso, Chile. Results: TEVAR was performed in 31 patients, male sex $74.2 \%$, average age 67.8 years (range $53-85$ ), symptomatic $64.5 \%$, associated pathologies: systemic arterial hypertension (77.4\%), smoking (67.7\%) and dyslipidemia (38.7\%), indications for endovascular repair were descending aortic aneurysm (51.61\%), complicated Stanford type B chronic aortic dissection (29.03\%), and complicated Stanford type B acute aortic dissection (19.35\%), neurological morbidity $(6.45 \%)$, cardiovascular morbidity $(12.9 \%)$, complications related to the stent $(29.03 \%)$, where endoleaks predominate (19.35\%), average hospital stay of 5.2 days (range 3 to 17), average followup of 47.3 months (9-108), primary technical success (100\%), survival rate at 1, 3, 5 years of $96.77 \%$, 93.54 and $90.32 \%$ respectively, there was no mortality less than 30 days or reoperation. Discussion: the evidence shows that TEVAR can be performed safely and effectively, meeting strict clinical criteria and anatomical conditions, which represents the modality of choice for the repair of lesions in the descending aorta. Conclusion: The results show that TEVAR represents an excellent therapeutic strategy, less invasive, with low associated morbidity and mortality.

Key words: TEVAR; thoracic aorta endoprosthesis; thoracic aortic aneurysm; aortic dissection.

\section{Resumen}

Introducción: Las diversas patologías de la aorta torácica descendente, representan una implícita amenaza para la vida, y son potencialmente tratables mediante reparación endovascular. Objetivo: Evaluar los resultados de la reparación endovascular de la aorta torácia descendente (TEVAR). Material y Método: Estudio observacional, retrospectivo y descriptivo, donde se analiza TEVAR, en un período de 10 años (2009 al 2019), en el Hospital Dr. Eduardo Pereira de Valparaíso, Chile. Resultados: Se realizó TEVAR en 31 pacientes, sexo masculino 74,2\%, femenino 25,8\%, edad promedio 67,8 años (rango 53-85), patologías asociadas: hipertensión arterial sistémica (77,4\%), tabaquismo $(67,7 \%)$ y dislipidemia $(38,7 \%)$, las indicaciones para TEVAR fueron: el aneurisma de la aorta descendente $(51,61 \%)$, la disección tipo B crónica complicada (29,03\%), y la disección tipo B aguda complicada (19,35\%), relacionado al procedimiento se evidenció: morbilidad cardiovascular (12,9\%) y morbilidad neurológica (6,45\%), complicaciones relacionadas a la endoprótesis (29,03\%), incidencia de endofugas (19,35\%), estancia hospitalaria promedio de 5,2 días (rango 3 a 17), seguimiento promedio de 47,3 meses (9-108), éxito técnico primario (100\%), tasa de reintervención: $3,22 \%$, tasa de supervivencia a 1, 3, 5 años del 96,77\%, 93,54 y 90,32\% respectivamente, no hubo mortalidad menor a 30 días. Discusión: La evidencia demuestra que TEVAR puede realizarse en forma segura y efectiva, cumpliendo estrictos criterios clínicos y condiciones anatómicas, representando la modalidad de elección para la reparación de las lesiones de la aorta descendente. Conclusión: Los resultados demuestran que TEVAR, representa una excelente estrategia terapeutica, menos invasiva, con baja morbilidad y mortalidad asociada.

Palabras clave: TEVAR; endoprótesis aorta torácica; aneurisma aorta torácica; disección aórtica.
'Servicio de Cirugía. Hospital Dr. Eduardo Pereira Ramírez. Valparaíso, Chile.

2Departamento de Cirugía Universidad de Valparaíso. Valparaíso, Chile.

Recibido 2020-05-18 y aceptado 2020-08-28

Correspondencia a: Dr. Luis C. Hernandez M. luiscesarh@gmail.com 


\section{Introducción}

Las diversas lesiones de la aorta torácica descendente (ATD), constituyen patologías potencialmente mortales, que incluyen un amplio espectro de enfermedades como el síndrome aórtico agudo, aneurismas, lesiones traumáticas, iatrogénia, inflamatorias y patologías congénitas ${ }^{1}$.

El síndrome aórtico agudo está constituido por tres entidades, la disección aórtica (DA), el hematoma intramural (HIM) y la úlcera penetrante aortica (UPA) ${ }^{2}$. Su incidencia es de 3 casos por cada 100.000 habitantes, de los cuales el $80 \%$ corresponden a DA, $15 \%$ HIM y $5 \%$ UPA $^{2,3}$.

Se denomina aneurisma de la aorta descendente (AAD), aquellos con diámetro $\geq 40 \mathrm{~mm}$, que se localizan en algún segmento entre la subclavia izquierda y el tronco celíaco ${ }^{4}$, su incidencia es de 6 a 10 casos por 100.000 habitantes, con mayor prevalencia entre la sexta y séptima década de la vida, y una relación 4:1 hombre/mujer ${ }^{4,5}$.

Los $\mathrm{AAD}$, presentan mayor incidencia de ruptura en comparación a los de aorta abdominal, el riesgo de ruptura a 5 años entre 4 y $6 \mathrm{~cm}$ es del $16 \%$, y del $31 \%$ para diámetros superiores a $6 \mathrm{~cm}^{5,6}$. Alrededor de un $25 \%$ de los pacientes con AAD son portadores de aneurismas abdominales, por esta razón es fundamental realizar una evaluación completa de la aorta ${ }^{6}$.

La reparación endovascular de la aorta torácica (TEVAR), presenta como finalidad implantar una endoprótesis que cubra la lesión del endotelio, logrando la descompresión del lumen falso y su trombosis, o aislar un aneurisma, reforzando la zona debilitada de la pared aortica evitando su ruptura ${ }^{7,8}$.

El propósito del presente estudio, es identificar las variables involucradas en la enfermedad de la aorta torácica descendente (ATD), y los resultados relacionados a TEVAR, en pacientes intervenidos en el Hospital Dr. Eduardo Pereira de Valparaíso, Chile.

\section{Material y Métodos}

Estudio observacional, retrospectivo y descriptivo, donde se analiza TEVAR, en un período de 10 años (2009 al 2019), en el Hospital Dr. Eduardo Pereira de Valparaíso, Chile. Los criterios definidos como indicación y contraindicación para TEVAR, se describen en la Tabla 1, los criterios técnicos para la instalación de la endoprotesis aórtica se describen en la Tabla 2.

Las variables principales del estudio corresponden a las complicaciones relacionadas a la endopro- tesis, supervivencia, reintervención, y morbilidad quirúrgica, con énfasis en la morbilidad cardiovascular, neurológica y el síndrome post implantación (SPI). Las variables secundarias estudiadas fueron sexo, edad, comorbilidades, síntomatología, sangrado, necesidad de transfusión y estancia hospitalaria.

Las endoprótesis utilizadas corresponden a las marcas comerciales Valiant ${ }^{\mathrm{TM}}$ (Medtronic, EE.UU), Zenith (TX2 ${ }^{\circledR}$, Alpha ${ }^{\mathrm{TM}}$; Cook Medical, EE.UU), Conformable GORE ${ }^{\circledR}$ TAG $^{\circledR}$ (Gore Medical, EE.UU), Relay Plus ${ }^{\circledR}$ (Bolton Medical, EE.UU).

Todas las intervenciones fueron efectuadas con anestesia general y monitorización invasiva de la presión arterial sistémica, el abordaje utilizado fue siempre la arteria femoral común. La liberación de las endoprótesis se realizó siempre con hipotensión controlada (presión arterial media (PAM) $\leq 70 \mathrm{~mm}$ $\mathrm{Hg}$ ), medida que fue posible administrando transitoriamente vasodilatadores endovenosos.

En casos seleccionados debido ausencia de un cuello proximal adecuado (sector sano de aorta $\geq 15 \mathrm{~mm}$ de longitud), y previo estudio mediante angiotomografía o angiografía selectiva de la arteria vertebral derecha, con el objetivo de confirmar su permeabilidad, y conformación de la circulación vertebrobasilar, se decidió cubrir intencionalmente el origen de la arteria subclavia izquierda (ASI), realizando cirugía de revascularización (puente carótido subclavio), en pacientes seleccionados.

Como estratégia de protección contra la isquemia medular, se aplicó en todos los pacientes dos medidas, la primera corresponde a la instalación de un cateter intradural, para permitir en caso de ser necesario el drenaje de líquido cefalorraquídeo (LCR), y la segunda medida consistió en mantener la presión de perfusión de la médula espinal $>80$ $\mathrm{mmHg}$, definida como la presión arterial media (PAM) - presión del LCR. Medida posible mediante la administración endovenosa de vasoactivos, logrando así mantener la PAM > $100 \mathrm{mmHg}$, y la presión del LCR entre 10-12 $\mathrm{mmHg}$, drenando un máximo de LCR de10cc/hora.

La remoción del cateter intradural se realizó siempre en las $72 \mathrm{~h}$ posteriores a su inserción, permaneciendo cerrado durante $12-18 \mathrm{~h}$ previas a su retiro, con la finalidad de evaluar la aparición de síntomas neurológicos. En caso de aparición de algún tipo de déficit neurológico, se administraron vasoactivos hasta lograr valores de PAM $>100 \mathrm{mmHg}$.

La vigilancia post operatoria de todos los pacientes, ocurrió en la Unidad de Paciente Crítico, garantizando un control estricto de la presión arterial, evaluando la actividad motora y sensitiva de las extremidades cada $6 \mathrm{~h}$, recibiendo todos los pa- 
Tabla 1. Indicaciones y contraindicaciones para TEVAR

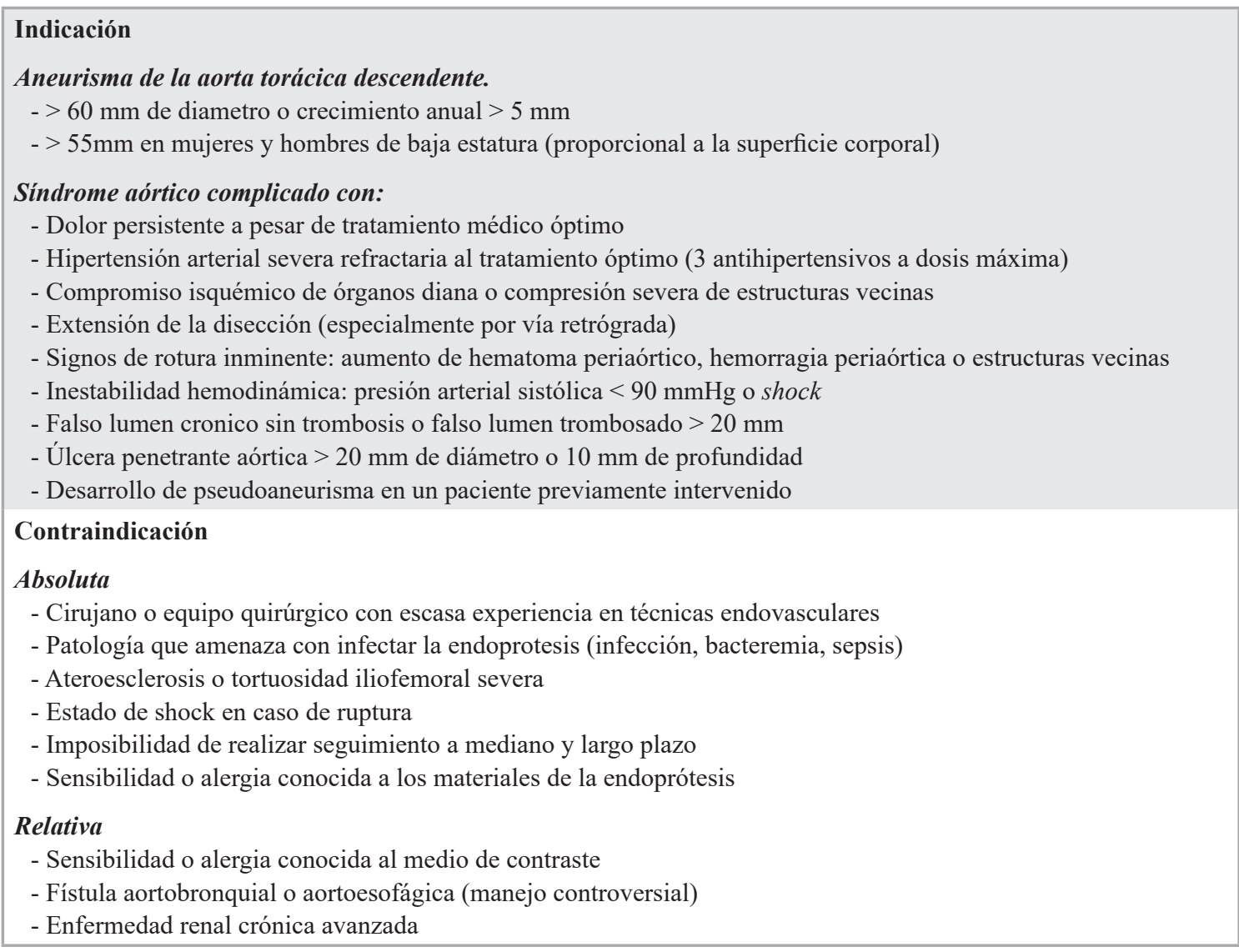

Tabla 2. Criterios técnicos para TEVAR

- Distancia del cuello proximal > $15 \mathrm{~mm}$ (desde el origen de la carótida común izquierda al borde proximal de la
endoprótesis), considerando ocluir la arteria subclavia izquierda en casos seleccionados
- Distancia del cuello distal > $15 \mathrm{~mm}$ (borde distal de la endoprótesis al origen del tronco celíaco)
- Diámetro de los cuellos proximal y distal deben medir entre $18 \mathrm{~mm}$ y $42 \mathrm{~mm}$
- Diámetro de la arteria femoral común o ilíaca externa $>7 \mathrm{~mm}$ (es posible acceder a la arteria ilíaca común por
disección retroperitoneal)
- Arterias permeables, sin trombos, con moderada angulación y calcificación
- Ausencia de calcificaciones y trombo mural en las áreas de implante de la endoprótesis

cientes diariamente y a permanencia, antiagregación plaquetaria con ácido acetilsalicílico $100 \mathrm{mg}$.

En todos los pacientes se realizó seguimiento clínico ambulatorio, y mediante angiotomografía torácoabdominal a los 1,6 y 12 meses durante el primer año, y posteriormente anual.

El análisis estadístico se llevó a cabo con el programa Stata versión 12, para verificar la asociación entre las variables más importantes se aplicó la prueba de Fisher, considerando un valor de $\mathrm{p} \leq 0,05$ como estadísticamente significativo.

\section{Resultados}

Se realizó TEVAR en 31 pacientes, hombres $(64,52 \%)$ y mujeres $(35,48 \%)$, edad promedio 67,8 años (rango 53-85), síntomáticos $(64,51 \%)$, predo- 
minando el dolor torácico o lumbar $(85,7 \%)$ y la hipertensión arterial (75\%). Asintómaticos $(35,49 \%)$, diagnosticados como hallazgo incidental al realizar una tomografía torácica, por causas en su mayoría oncológicas $(63,6 \%)$.

Las comorbilidades asociadas se describen en la Tabla 3, predominando la hipertensión arterial $(77,4 \%)$, tabaquismo $(67,7 \%)$ y dislipidemia $(38,7 \%)$. EL 25,80\% de los casos, presentó aneurismas arteriales asociados, de estos el $50 \%$ en la aorta abdominal infrarenal, iliaca común $(25 \%)$, arteria hipogástrica $(12,5 \%)$ y arteria poplítea $(12,5 \%)$.

Las indicaciónes para TEVAR se describen en la Tabla 4, donde predomina el AAD $(51,61 \%)$, la disección crónica Stanford B complicada (29,03\%), la disección aguda o subaguda Stanford B complicada (19,35\%). Realizando TEVAR programado en el $90,32 \%$ y urgente en el $9,68 \%$.

Tabla 3. Patologías asociadas en 31 pacientes con patología de la aorta torácica descendente

\begin{tabular}{|lrc|}
\hline Comorbilidad & n & \% \\
Hipertensión arterial & 23 & 74,19 \\
Tabaquismo & 21 & 67,74 \\
\hline Dislipidemia & 12 & 38,70 \\
Obesidad (IMC > 30) & 8 & 25,80 \\
Diabetes mellitus & 7 & 22,58 \\
EPOC & 5 & 16,13 \\
Cardiopatía coronaria & 5 & 16,13 \\
\hline Enfermedad renal avanzada & 3 & 9,67 \\
\hline Colagenopatía & 2 & 6,45 \\
\hline
\end{tabular}

Tabla 4. Indicación de TEVAR en 31 pacientes

\begin{tabular}{|lrr|}
\hline Indicación & $\mathbf{n}$ & $\mathbf{\%}$ \\
\hline Aneurisma de la aorta torácica descendente & 16 & 51,61 \\
- > 60 mm en hombres o 55 mm en mujeres & 14 & 45,15 \\
- Rápido crecimiento (> 5 mm/anual) & 2 & 6,45 \\
Disección crónica Stanford B complicada & 9 & 29,03 \\
- Dilatación aneurismatica > 50 mm & 5 & 16,12 \\
- Úlcera penetrante aórtica > 20 mm de diámetro & 4 & 12,90 \\
Disección aguda o subaguda Stanford B complicada & 6 & 19,35 \\
- Dolor persistente a pesar de tratamiento médico óptimo & 2 & 6,45 \\
- Hipertensión severa refractaria al tratamiento & 1 & 3,22 \\
- Hemorragia periaórtica o a estructuras vecina & 1 & 3,22 \\
- Compromiso isquémico de órganos diana & 1 & 3,22 \\
- Extensión de la disección hacia distal & 1 & 3,22 \\
\hline
\end{tabular}

El éxito técnico primario, definido como el despliegue adecuado de la endoprotesis, sin endofuga tipo I o III, ausencia de conversión quirúrgica o muerte en las primeras $24 \mathrm{~h}$, fue del 100\%. Las complicaciones relacionadas al TEVAR se describen en la Tabla 5, evidenciando una morbilidad cardiovascular del $12,9 \%$, infecciosa $(9,67 \%)$, local $(9,67 \%)$ y neurológica $(6,45 \%)$.

Se realizó la oclusión intencional de la ASI en un $22,58 \%$, el $57,14 \%$ de estos sin cirugía de revascularización preventiva de la ASI, no evidenciando morbililidad neurológica asociada, ni claudicación de la extremidad superior.

La incidencia de complicaciones relacionadas a la implantación de la endoprótesis aórtica fue del $29,03 \%$, determinada por las endofugas $(19,35 \%)$, dilatación del aneurisma $(3,22 \%)$, embolización $(3,22 \%)$ y fractura de la endoprótesis $(3,22 \%)$, sin evidenciar disección retrograda, migración o trombosis (Tabla 6). Presentando las endofugas la siguiente distribución: tipo I (16,67\%), II (83,3\%), III, IV y V $(0 \%)$.

El único caso con endofuga tipo I (subtipo Ib), presentó manifestación tardía a los 12 meses del post operatorio, condicionando a la dilatación del $20 \%$ del saco aneurismático, ameritando la reintervención exitosa mediante la implantación de un stent. En todas las endofugas tipo 2 se realizó

Tabla 5. Complicaciones del TEVAR en 31 pacientes

\begin{tabular}{|llc|}
\hline Complicación & $\mathbf{n}$ & $\mathbf{\%}$ \\
Síndrome post implantación & 5 & 16,12 \\
Cardiovascular & 4 & 12,9 \\
Arritmias & 2 & 6,45 \\
Infarto agudo al miocardio & 1 & 3,22 \\
Angina inestable & 1 & 3,22 \\
Infecciosa & 3 & 9,67 \\
Neumonía intra hospitalaria & 1 & 3,22 \\
Infección endoprótesis & 1 & 3,22 \\
Infeccción del tracto urinario & 1 & 3,22 \\
Local & 3 & 9,67 \\
Pseudoaneurisma de la arteria femoral común & 1 & 3,22 \\
Hematomia inguinal & 1 & 3,22 \\
Linforrea & 1 & 3,22 \\
Neurológica & 2 & 6,45 \\
Paraparesia transitoria & 1 & 3,22 \\
Ictus isquémico transitorio & 1 & 3,22 \\
\hline Deterioro de la función renal & 1 & 3,22 \\
\hline Ventilación mecánica invasiva prolongada & 1 & 3,22 \\
\hline
\end{tabular}


Tabla 6. Complicaciónes relacionadas a la implantación de la endoprótesis

\begin{tabular}{|c|c|c|c|c|c|c|c|c|c|}
\hline $\begin{array}{l}\text { Endoprotesis } \\
\text { utilizada }\end{array}$ & n (\%) & $\begin{array}{c}\text { Endofuga } \\
\text { n (\%) }\end{array}$ & $\begin{array}{c}\text { Migración } \\
\text { n (\%) }\end{array}$ & $\begin{array}{c}\text { Fractura } \\
\text { n }(\%)\end{array}$ & $\begin{array}{c}\text { Disección } \\
\text { n (\%) }\end{array}$ & $\begin{array}{c}\text { Dilatación } \\
\text { n (\%) }\end{array}$ & $\begin{array}{c}\text { Trombosis } \\
\text { n (\%) }\end{array}$ & $\begin{array}{c}\text { Embolización } \\
\text { n (\%) }\end{array}$ & $\begin{array}{l}\text { Total } \\
\text { n (\%) }\end{array}$ \\
\hline Zenith TX2 ${ }^{\circledR}$ & $10 / 31(32,35)$ & $2 / 10(20)$ & 0 & 0 & 0 & $1 / 10(10)$ & 0 & 0 & $3 / 10(30)$ \\
\hline Zenith Alpha ${ }^{\mathrm{TM}}$ & $8 / 31(25,80)$ & $2 / 8(25)$ & 0 & 0 & 0 & 0 & 0 & 0 & $2 / 8(25)$ \\
\hline Valiant $^{\mathrm{TM}}$ & $6 / 31(19,35)$ & $1 / 6(16,67)$ & 0 & 0 & 0 & & 0 & $1 / 6(16,67)$ & $2 / 6(33,33)$ \\
\hline $\begin{array}{l}\text { Conformable } \\
\text { GORE }^{\circledR} T^{-1} G^{\circledR}\end{array}$ & $4 / 31(12,90)$ & $1 / 4(25)$ & 0 & 0 & 0 & 0 & 0 & 0 & $1 / 4(25)$ \\
\hline Relay Plus ${ }^{\circledR}$ & $3 / 31(9,67)$ & 0 & 0 & $1 / 3(33,33)$ & 0 & 0 & 0 & 0 & $1 / 3(33,33)$ \\
\hline Total & 31 (100) & $6 / 31(19,35)$ & $0 \%$ & $1 / 31(3,22)$ & $0 \%$ & $1 / 31(3,22)$ & $0 \%$ & $1 / 31(3,22)$ & $9 / 31(29,03)$ \\
\hline
\end{tabular}

manejo expectante, presentando todas resolución espontánea en los 6 meses posteriores al TEVAR, sin evidenciar complicaciones asociadas.

Un caso $(3,22 \%)$ presentó fractura focal de la endoprótesis, realizandose manejo conservador sin evidenciar complicaciones asocidas, hasta el fallecimiento del paciente a los 45 meses del seguimiento debido a cardiopatía coronaria. Un caso $(3,22 \%)$ presentó embolización de placas de ateroma a ramas de la arteria hipogástrica izquierda, evidenciado por la presencia de dolor y claudicación glútea leve, confirmando el hallazgo mediante angiotomografía.

La incidencia de complicaciones neurológicas fue del $6,25 \%$, determinada por una caso $(3,22 \%)$ de ictus isquémico transitorio, en las primeras horas del post operatorio, sin presentar alteración en la angiotomografía, ni deficit neurológico a largo plazo, y un caso $(3,22 \%)$ de paraparesia transitoria, durante el primer día de post operatorio, manejada medicamente con medidas de protección medular, evolucionando satisfactoriamente sin deficit motor ni sensitivo a largo plazo.

Un caso $(3,22 \%)$ presentó infección de la endoprotesis, diagnosticada el día 23 de post operatorio, confirmada mediante hemocultivos periféricos sucesivos, aislando Staphylococcus spp, recibiendo antibioticoterapia endovenosa de amplio expectro, presentando evolución catastrófica con el fallecimiento del paciente, al día 40 de post operatorio debido a sepsis.

La incidencia del síndrome post implantación (SPI) fue del 16,12\%, diagnosticando todos los casos en las primeras $48 \mathrm{~h}$ del post operatorio, caracterizados por fiebre $\geq 38^{\circ} \mathrm{C}$, leucocitosis $\geq 15,000 \times 10^{3} /$ $\mathrm{mm}^{3}$ y PCR $\geq 100 \mathrm{mg} / \mathrm{L}$. Descartandose patología infecciosa mediante estudios de imagen y cultivos seriados negativos.

El sangrado promedio relacionado al procedimiento fue de $195 \mathrm{cc}$, ameritando en el $6,45 \%$ de los casos transfusión promedio de 2 unidades de globulos rojos. La estancia hospitalaria promedio fue de 5,28 días (rango 3 a 17), con un seguimiento promedio de 47,3 meses (9-108).

No hubo mortalidad a los 30 días, ni mortalidad relacionada al procedimiento, con una tasa de reintervención del $3,22 \%$, y tasa de supervivencia a 1, 3, 5 años del 96,77\%, 93,54 y 90,32\% respectivamente.

\section{Discusión}

La mortalidad operatoria precoz de la cirugía abierta en centros de excelencia, se reporta entre un 8 y $20 \%$ para procedimientos electivos, y de hasta un $60 \%$ en procedimientos de urgencia ${ }^{9}$, con una morbilidad cercana al 50\% relacionada principalmante a causas cardiovasculares, respiratorias, renales e isquemia medular ${ }^{9,10}$.

TEVAR se ha constituido como el procedimiento de elección en casos seleccionados, al ser menos invasivo frente a la cirugía convencional, con una mortalidad precoz del 3 al $8,5 \%$ y a 5 años del $13 \%$ al $28 \%^{10,11}$. No evidenciando en nuestra serie mortalidad precoz, con una mortalidad a 5 años del $9,32 \%$.

La revascularización preventiva de la ASI, esta indicada en pacientes con un polígono de Willis incompleto, arteria vertebral izquierda dominante, brazo izquierdo dominante, enfermedad obstructiva de la arteria vertebral derecha o carotídea bilateral, bypass mamario-coronario izquierdo, fístula de diálisis del brazo izquierdo, variantes anatómicas del cayado aórtico (subclavia derecha aberrante) o compromiso extenso de la aorta toracoabdominal ${ }^{12-14}$.

En nuestro estudio fue posible la oclusión intencional de la ASI, sin cirugía de revascularización preventiva en el $12,90 \%$, sin evidenciar morbililidad 
neurológica asociada, ni claudicación de la extremidad superior.

La incidencia de paraplejía/paraparesia posterior a TEVAR es del 3,4\% ${ }^{15}$, el recubrimiento aórtico $\geq 20 \mathrm{~cm}$, la oclusión de intercostales a nivel T10, el recubrimiento de la subclavia izquierda, y el flujo comprometido de arterias hipogástricas, aumentan el riesgo de complicaciones neurológicas ${ }^{16,17}$. En nuestra serie solo se evidenció un caso $(3,22 \%)$ de paraparesia transitoria, en un paciente con recubrimiento extenso de la aorta $(20 \mathrm{~cm})$, que involucro nivel T10.

El síndrome postimplantación (SPI) es un trastorno transitorio, producido en el posoperatorio inmediato posterior a la implantación de una endoprótesis aórtica, caracterizado por la presencia de anorexia, dolor torácico o lumbar, leucocitosis, fiebre, elevación de la proteína $\mathrm{C}$ reactiva, ocasionalmente asociado a disminución del recuento plaquetario o alteración de la coagulación ${ }^{18}$. La interacción entre la endoprótesis, el endotelio vascular y la adhesión del trombo, generan la liberación de citocinas, metabolitos del ácido araquidónico y activación leucocitaria, ocasionando los síntomas sistémicos ${ }^{18,19}$.

La respuesta inflamatoria severa es capaz de generar una extensa lesión endotelial, debido al efecto directo de los agentes mediadores, conduciendo a complicaciones graves como disfunción pulmonar, eventos cardiovasculares, insuficiencia renal y disfunción multiorgánica ${ }^{20}$. Los pacientes con diagnóstico de SPI presentan una probabilidad 4 veces mayor de sufrir un evento cardiovascular o evento adverso, por cada día adicional de fiebre la posibilidad de un episodio cardiovascular aumenta en un $67,9 \%{ }^{21}$, y por cada aumento de 10 unidades de la PCR, esta probabilidad aumenta en un $15 \% \%^{21,22}$.

En nuestra serie la incidencia de SPI fue del $16,12 \%$, donde los factores de riesgo asociados fueron el recubrimiento aórtico $\geq 15 \mathrm{~cm}$, uso de endoprótesis cubiertas por poliéster, edad $\leq 75$ años y tiempo quirúrgico $>180 \mathrm{~min},(\mathrm{p}<0,05)$. Presentando las endoprótesis Zenith TX2 ${ }^{\circledR}$ y Zenith Alpha ${ }^{\mathrm{TM}}$, la mayor incidencia de SPI con $22,2 \%$ y $20 \%$ respectivamente (ambas endoprótesis recubiertas por poliester).

El accidente vascular encefálico (AVE) en el TEVAR presenta una incidencia del 3,1 al $5 \%{ }^{23}$, y se encuentra relacionado a factores sistémicos (hipotensión, hipertensión, anticoagulación), causas intracraneales (estenosis de vasos cerebrales, hemorragia, edema, drenaje súbito de líquido cefalorraquídeo), embolía (aire, ateroma, trombo), implante $\geq 3$ stent, recubrimiento de la subclavia, insuficiencia renal y reparación simultánea de aneurisma abdominal ${ }^{22,24}$.
La ateromatosis generalizada y duración del procedimiento superior a $160 \mathrm{~min}$, se asocian con un riesgo incrementado de AVE, relacionado con la manipulación de guías, catéteres y dispositivos en el arco aórtico ${ }^{25}$. Nuestra incidencia fue del $3,22 \%$ (un caso), en un paciente que presentó un ictus isquémico transitorio, cuyos factores de riesgo fueron ateromatosis generalizada, aneurisma de gran tamaño $(6 \mathrm{~cm})$, procedimiento prolongado $(180 \mathrm{~min})$ e hipotensión prolongada durante el procedimiento.

Las endofugas son la complicación más frecuente, con una incidencia del $35 \%$ para las series estadounidenses $^{25,26}$ y $17 \%$ en el Eurostar ${ }^{27}$; como factores de riesgo independiente para endofugas primarias, se encuentran el sexo femenino, edad $\geq 75$ años y diámetro del aneurisma $>60 \mathrm{~mm}^{28}$. Nuestra incidencia fue del $19,35 \%$, evidenciando como factor de riesgo en el $83,3 \%$ la presencia de un aneurisma $>60 \mathrm{~mm}$.

Si en el control posterior al TEVAR, se evidencia ausencia de endofugas y disminución del tamaño del aneurisma, el tratamiento se considera satisfactorio. Si el saco aneurismático aumenta de tamaño, debe descartarse la presencia de endofugas ${ }^{29}$.

La infección de la endoprótesis en TEVAR, presenta una incidencia del $1,5 \%$ al $4,8 \%{ }^{30}$, la causa más frecuente es la contaminación perioperatoria, seguida de cirugía de urgencia, diseminación hematógena, reintervenciones, inmunodeficiencia, y desarrollo de fístulas aortobronquiales o aortoesofágicas $^{30,31}$.

Los síntomas más frecuentes son dolor torácico o lumbar, descrito entre el $44 \%$ y $75 \%$, fiebre entre el $33 \%$ y $81 \%$, síndrome constitucional y fenómenos isquémicos por embolia séptica o sepsis ${ }^{31,32}$. En nuestro estudio la incidencia fue del $3,22 \%$ (un caso), donde los factores de riesgo asociados fueron diabetes mellitus, cirugía de urgencia y recubrimiento aórtico extenso $(20 \mathrm{~cm})$, los síntomas presentados por el paciente fueron fiebre, dolor torácico e hipertensión arterial severa.

Nuestros resultados reproducen con gran exactitud los hallazgos reportados en la literatura, sin embargo presentan como limitante el ser un análisis retrospectivo unicéntrico. Es importante realizar estúdios prospectivos aleatorizados multicéntricos que evaluen TEVAR, y estudios que comparen los resultados de la cirugía abierta frente a TEVAR.

\section{Conclusión}

La utilización de estrictos criterios de selección, permite la oclusión intencional de la ASI, sin evi- 
denciar complicaciones clínicas, ni neurológicas asociadas a largo plazo.

La implantación urgente de una endoprótesis aórtica y las reintervenciones, se relacionan con un mayor riesgo de infección, donde la mortalidad es elevada especialmente en pacientes inestables, el tratamiento no está estandarizado, resultando una decisión compleja y urgente.

Los resultados demuestran que TEVAR, representa una excelente alternativa terapeutica, menos invasiva, con baja morbilidad y mortalidad asociada. Es importante realizar estudios prospectivos que evaluen a largo plazo la durabilidad, costo eficiencia y complicaciones relacionadas al procedimiento.

\section{Responsabilidades éticas}

Protección de personas y animales. Los autores declaran que para esta investigación no se han realizado experimentos en seres humanos ni en animales.

Confidencialidad de los datos. Los autores declaran que en este artículo no aparecen datos de pacientes.

Conflictos de interés: no hay.

\section{Bibliografía}

1. Erbel R, Aboyas V, Boileau C, Bossone E, Di Bartolomeo R, Eggebrecht H, et al. Guía ESC 2014 sobre diagnóstico y tratamiento de la patología de la aorta. Rev Esp Cardiol. 2015;68:242.e1-e69.

2. Lansman S, Saunders P, Malekan R, Spielvogel D. Acute aortic syndrome. J Thorac Cardiovasc Surg. 2010;140: S927.

3. Vega J, Zamorano J, Pereira N, Galleguillos A. Síndrome aórtico agudo: Revisión de la literatura y actualización del tema. Rev Med Chile 2014;142:34452.

4. Erbel R, Aboyans V, Boileau C, Bossone E, Di Bartolomeo R, Evangelista A, et al. 2014 ESC Guidelines on the diagnosis and treatment of aortic diseases: Document covering acute and chronic aortic diseases of the thoracic and abdominal aorta of the adult. Eur Heart J. 2014;35:2873-926.

5. Jovin IS, Duggal M, Ebisu K, Paek $\mathrm{H}$, Oprea AD, Tranquilli M, et al. Comparison of the effect on long term outcomes in patients with thoracic aortic aneurysms of taking versus not taking a statin drug. Am J Cardiol. 2012;109:10504.

6. Girardi LN, Krieger KH, Altorki NK, Mack CA, Lee LY, Isom OW. Ruptured descending and thoracoabdominal aortic aneurysms. Ann Thorac Surg. 2002;74:1066-70.

7. Estrera AL, Jan A, Sandhu H, Shalhub S, Medina-Castro M, Nguyen TC, et al. Outcomes of open repair for chronic descending thoracic aortic dissection. Ann Thorac Surg. 2015;99:786-93.

8. Grabenwoger, M, Alfonso, F, Bachet J, Bonser R, Czerny M, Eggebrecht, $\mathrm{H}$, et al Thoracic Endovascular Aortic Repair (TEVAR) for the treatment of aortic diseases: a position statement from the European Association for Cardio-Thoracic Surgery (EACTS) and the European Society of Cardiology (ESC), in collaboration with the European Association of Percutaneous Cardiovascular Interventions (EAPCI). European Heart Journal 2012;33:1558-63.

9. Desai ND, Burtch K, Moser W, Moeller P, Szeto WY, Pochettino A, et al. Longterm comparison of thoracic endovascular aortic repair (TEVAR) to open surgery for the treatment of thoracic aortic aneurysms. J Thorac Cardiovasc Surg. 2012;144:6049; discussion 609-11.

10. Cheng D, Martin J, Shennib H, Dunning J, Muneretto C, Schueler S, et al. Endovascular aortic repair versus open surgical repair for descending thoracic aortic disease a systematic review and meta-analysis of comparative studies. J Am Coll Cardiol. 2010;55:986-1001.

11. Patel HJ, Williams DM, Upchurch GR Jr, Dasika NL, Deeb GM. A comparative analysis of open and endovascular repair for the ruptured descending thoracic aorta. J Vasc Surg. 2009;50:1265-70.

12. Dunning J, Martin JE, Shennib H, Cheng DC. Is it safe to cover the left subclavian artery when placing an endovascular stent in the descending thoracic aorta? Interact Cardiovasc Thorac Surg. 2008;7:690-7.

13. Matsumura JS, Lee WA, Mitchell RS,
Farber MA, Murad MH, Lumsden AB, et al. Society for Vascular Surgery. The Society for Vascular Surgery Practice Guidelines: management of the left subclavian artery with thoracic endovascular aortic repair. J Vasc Surg. 2009;50:1155-8.

14. Cheng D, Martin J, Shennib H, Dunning J, Muneretto C, Schueler S, et al. Endovascular aortic repair versus open surgical repair for descending thoracic aortic disease a systematic review and meta-analysis of comparative studies. J Am Coll Cardiol. 2010;55:986-1001.

15. Riesenman PJ, Farber MA, Mendes RR, Marston WA, Fulton JJ, Keagy BA. Coverage of the left subclavian artery during thoracic endovascular aortic repair. J Vasc Surg. 2007;45:90-4.

16. Buth J, Harris PL, Hobo R, van Eps R, Cuypers P, Duijm L, et al. Neurologic complications associated with endovascular repair of thoracic aortic pathology: Incidence and risk factors. a study from the European Collaborators on Stent/Graft Techniques for Aortic Aneurysm Repair (EUROSTAR) registry. J Vasc Surg. 2007;46:1103-1110; discussion 1110-1.

17. Khoynezhad A, Donayre CE, Bui H, Kopchok GE, Walot I, White RA. Risk factors of neurologic deficit after thoracic aortic endografting. Ann Thorac Surg. 2007;83(Sup- pl):882-9.

18. Velázquez OC, Carpenter JP, Baum RA, Barker CF, Golden M, Criado F, et al. Perigraft air, fever, and leukocytosis after endovascular repair of abdominal aortic aneurysms. Am J Surg. 1999;178:185-9. 
19. Froon AH, Greve JW, Van der Linden CJ, Buurman WA. Increased concentrations of cytokines and adhesion molecules in patients after repair of abdominal aortic aneurysm. Eur J Surg. 1996;162:287-96.

20. Gerasimidis T, Sfyroeras G, Trellopoulos G, Skoura L, Papazoglou $\mathrm{K}$, Konstantinidis K, et al. Impact of endograft material on the inflammatory response after elective endovascular abdominal aortic aneurysm repair. Angiology 2005;56:743-53.

21. Arnaoutoglou E, Kouvelos G, Papa N, Kallinteri A, Milionis H, Koulouras V, et al. Prospective Evaluation of Postimplantation Inflammatory Response After EVAR for AAA: Influence on Patients' 30 Days Outcome. European Journal of Vascular and Endovascular Surgery 2014; epub ahead(2015):1-9.

22. Kwon H, Ko GY, Kim MJ, Han Y, Noh M, Kwon TW, et al. Effects of postimplantation systemic inflammatory response on long-term clinical outcomes after endovascular aneurysm repair of an abdominal aortic aneurysm. Medicine (Baltimore) 2016;95:e4532

23. Khoynezhad A, Donayre CE, Bui H,
Kopchok GE, Walot I, White RA. Risk factors of neurologic deficit after thoracic aortic endografting. Ann Thorac Surg. 2007;83(Sup- pl):882-9.

24. Feezor RJ, Martin TD, Hess PJ, Klodell CT, Beaver TM, Huber TS, et al. Risk factors for perioperative stroke during thoracic endovascular aortic repairs (TEVAR). J Endovasc Ther. 2007;14:56873.

25. Morales JP, Greenberg RK, Lu Q, Cury M, Hernandez AV, Mohabbat W, et al. Endoleaks following endovascular repair of thoracic aortic aneurysm: etiology and outcomes. J Endovasc Ther. 2008;15:631-8.

26. Alsac JM, Khantalin I, Julia P, Achouh P, Farahmand P, Capdevila C, et al. The significance of endoleaks in thoracic endovascular aneurysm repair. Ann Vasc Surg. 2011;25:345-51.

27. Buth J, Harris PL, Hobo R, van Eps R, Cuypers P, Duijm L, et al. Neurologic complications associated with endovascular repair of thoracic aortic pathology: Incidence and risk factors. a study from the European Collaborators on Stent/Graft Techniques for Aortic Aneurysm Repair (EUROSTAR) registry.
J Vasc Surg. 2007;46:1103-10; discussion 1110-1.

28. Castejón A, Reyes M, Garnica M, Romero J, Ocaña C, Gandarias Z. Clínica, diagnóstico, tratamiento y evolución de la infección de las endoprótesis aórticas del sector torácico y abdominal. Angiología 2016;68:359-452.

29. Sommer W, Becker C, Haack M, Rubin G, Weidenhagen R, Nikolaou K, et al. "Time-resolved CT angiography for the Detection and classification of endoleaks." Radiology 2012;263:917-26.

30. Davila VJ, Stone W, Duncan AA, Wood E, Jordan WD, Zea N, et al. A multicenter experience with the surgical treatment of infected abdominal aortic endografts. J Vasc Surg. 2015;62: 877-83.

31. Laser A, Barker N, Rectenwald J, Eliason JL, Criado-Pallares E, Upchurch GR. Graft infection after endovascular abdominal aortic aneurysm repair. J Vasc Surg. 2011;54:58-63.

32. Lyons OT, Patel AS, Saha P, Clough RE, Price N, Taylor PR. A 14-year experience with aortic endograft infection: management and results. Eur J Vasc Endovasc Surg. 2013;46:306-13. 